\title{
Relevance and Incidence of Musculoskeletal Injuries in Indian Tennis Players; an Epidemiological Study
}

\author{
Shaji John Kachanathu', ${ }^{1, *}$ Parveen Kumar ${ }^{2}$, Mimansa Malhotra ${ }^{2}$ \\ ${ }^{1}$ College of Applied Medical Sciences, King Saud University, Riyadh, Saudi Arabia \\ ${ }^{2}$ Institute of Health and Rehabilitation Sciences, Indian Spinal Injury Center, New Delhi, India \\ *Corresponding author: johnsphysio@gmail.com
}

Received May 25, 2014; Revised June 18, 2014; Accepted July 24, 2014

\begin{abstract}
Professional tennis sports involve powerful movements repeatedly subjecting the musculoskeletal system to heavy mechanical load, thereby increasing risk for most acute and overuse injuries. Despite many researches in sports injuries, however, none of them has dealt with prevalence, incidence, and pattern of tennis injuries among Indian tennis players. The aim of this study was to prospectively make a survey of prevalence and incidence of musculoskeletal injuries in Indian tennis players. A convenience sample of 350 professional tennis players from various national tennis sports complexes participated in this study. A sample size of 256 with a mean age of $22.67 \pm 9.34$ years was compiled as per inclusion criteria. These included 173 males ( $24.23 \pm 10.20$ years) and 83 females (19.41 \pm 6.09 years). An Unpaired t-test and ANOVA test were used to compare between injury incidences in different epidemiological study groups. Overall Injury Incidence was 2.18 / 1000 playing hours and Prevalence was 15.62 / 100 tennis players. Elbow was the most commonly injured joint, followed wrist, ankle, shoulder, knee, calf, thigh and foot in decreasing order of their occurrence. The gender difference was insignificant. Tennis players sustain more overuse injuries in upper limbs and more acute injuries in lower limbs. The backhand was the most injury aggravating strokes for elbow injuries, for wrist it was forehand stroke. This study helps to understand the prevalence and incidence of musculoskeletal injuries among Indian tennis players. The findings also reinforce the need for continuing scientific professional training and preventive fitness measures of the weak areas to reduce musculoskeletal injuries.
\end{abstract}

Keywords: tennis injury, tennis survey, incidence, prevalence, tennis injury pattern

Cite This Article: Shaji John Kachanathu, Parveen Kumar, and Mimansa Malhotra, "Relevance and Incidence of Musculoskeletal Injuries in Indian Tennis Players; an Epidemiological Study." American Journal of Sports Science and Medicine, vol. 2, no. 5A (2014): 1-5. doi: 10.12691/ajssm-2-5A-1.

\section{Introduction}

Tennis is a popular global sport that attracts individuals from different age groups and with participation in more than 200 countries affiliated with the International Tennis Federation (Pluim et al. 2006). The game of tennis has evolved from the wooden-racket era of long, crafty points based on style and finesse, to the current fast paced, explosive sport based on power, strength and speed, where $210 \mathrm{~km} / \mathrm{h}$ serves are common. This evolution over the last 20 years has led to an increased interest in tennis research (Kavocs, 2007). Tennis, in its present form was conceived in England in the 1870s. In the 1880s, the British Army and Civilian officers brought the game to India. Soon after, regular tournaments like 'Punjab Lawn tennis Championship' at Lahore (Now in Pakistan) (1885); 'Bengal Lawn Tennis Championship' at Calcutta (1887) and the All India Tennis Championships in Allahabad in (1910) were organized (AITA, 2010). For the last 10 years, tennis practice has grown significantly for both recreation and competition purposes.
Sports injuries rank second highest in terms of cause of injury, after home and leisure accidents; and rank third in terms of severity, after traffic accidents and violence (Dekker et al. 2003). In recent years, more and more athletes are undertaking intense training at younger ages or participating in multiple sports in one season, thereby exposing themselves to more opportunities for acute injury and increasing their risk for overuse injuries. Injuries are often considered an inevitable part of sports. However, like other injuries, sports injuries are potentially avoidable (Adirim and Cheng, 2003). In spite of the positive effects that tennis practice has shown on physical and mental fitness, the increased number of tournaments and competitions determines an intense dedication to the training of young players. This intense practice exposes players to overtraining and excessive loads of specialized physical activity (Alberto et al. 2009). Modern professional tennis involves powerful movements repeatedly subjecting the musculoskeletal system to heavy mechanical load (Maquirriain and Ghisi, 2006).

Tennis coaches and instructors have several ways to teach tennis strokes depending on the age, level of playing, and ambitions of the player; furthermore, players choose different grips and personalize the movement (Alberto, 
2009). Court surface may play a role in injury rates and patterns. Different court surfaces can alter the demands that are placed on the tennis player. There are no specific data correlating injury to court surface (Kibler and Safran, 2005).

The results of epidemiological studies in tennis players have shown some variability. However, they all seem to identify a certain pattern of injury with respect to the location and type of injury (Bylak and Hutchinson, 1998). Data obtained from epidemiological studies of sports injuries are an essential requirement for developing injury prevention, treatment and rehabilitation strategies. In particular, epidemiological studies provide data required for the development, application and assessment of injury causation (Brooks and Fuller, 2006).

Despite many researches till date, none of the research has dealt with prevalence, incidence, and pattern of tennis injuries among Indian tennis players. This study was aimed at finding the pattern, prevalence and incidence of injuries in Indian tennis players by comparing against their age, gender, tennis experience, type of court used, skill level, gripping style, etc. None of the studies had correlated these many factors with the injuries in Indian tennis players.

\section{Materials and Methods}

Questionnaire: The tennis injury standard questionnaire was designed, which started with a consent form and instructions. The questionnaire had three parts, first part of the questionnaire included questions regarding the players' demographics, tennis history, and warm-up durations. Second part was consisted of questions relating to injury history. Only those players who were out of the game for 7 or more days due to any tennis related injury in the past 1 year were to answer this part of the questionnaire. Others were made to skip this part. Third part of the questionnaire consisted of gripping style of the players. A pilot study was done prior to final survey on a sample questionnaire with 10 players including 4 tennis coaches. They were able to understand and respond to the questionnaire. Only those players willing to participate voluntarily in this study were made to fill the questionnaire. If there was any problem in understanding any part of the questionnaire, the researcher and/or coaches were available to help explain the same to the subject. The present study was reviewed and approved by the institutional ethical committee and informed written consent form obtained prior to the study for all subjects.

Subjects: During this study a total of 350 questionnaires were distributed to 350 tennis players of different age groups over a period of 6 months at various national tennis sports complexes. Out of them 258 subjects returned the answered questionnaires and two of them were not fully answered. A sample size of 256 with a mean age of $22.67 \pm 9.34$ years was compiled as per inclusion criteria. A total of 256 included 173 males (24.23 \pm 10.20 years) and 83 females (19.41 \pm 6.09 years) and their questionnaires were used for analysis in the study.

Data Analysis: Nominal values were assigned to each nominal variable of the questionnaire. Incidence was calculated keeping the individual player as the unit of analysis. Statistical analysis was performed by using SPSS version 16 (IBM Corporation, USA) for Windows (Microsoft Corporation, USA). Unpaired t-test and ANOVA test were used to compare between injury incidences in different epidemiological study groups.

\section{Results}

A total of 256 with a mean age of $22.67 \pm 9.34$ years questionnaires were compiled analyzed. Sample size included 173 males and 83 females with mean age of $24.23 \pm 10.20$ and $19.41 \pm 6.09$ years respectively. The following results were categorized in different epidemiological study groups (Table 1).

Table 1.

\begin{tabular}{|c|c|c|c|c|}
\hline Study Groups & Number & $\%$ & Incidence & Prevalence \\
\hline Total & 256 & 100 & 2.18 & 15.62 \\
\hline \multicolumn{5}{|l|}{ Gender } \\
\hline Male & 173.00 & 67.58 & 2.29 & 16.76 \\
\hline Female & 83.00 & 32.42 & 1.91 & 13.25 \\
\hline \multicolumn{5}{|l|}{ Age group } \\
\hline Group 1 ( $<16$ yrs) & 63.00 & 24.61 & 2.35 & 6.35 \\
\hline Group 2 (16 - 26 yrs) & 100.00 & 39.06 & 2.01 & 16.00 \\
\hline Group 3 (> 266 yrs) & 93.00 & 36.33 & 2.31 & 21.50 \\
\hline \multicolumn{5}{|l|}{ ITN ranking group } \\
\hline Group $1(\mathrm{ITN}=1,2,3)$ & 45.00 & 17.58 & 1.98 & 42.22 \\
\hline Group $2(\mathrm{ITN}=4,5,6)$ & 139.00 & 54.30 & 2.26 & 13.67 \\
\hline Group $3(\mathrm{ITN}=7,8,9,10)$ & 72.00 & 28.12 & 2.94 & 2.78 \\
\hline \multicolumn{5}{|l|}{ Experience group } \\
\hline Group 1 ( $\leq 5$ yrs) & 154.00 & 60.16 & 2.70 & 6.50 \\
\hline Group 2 (6 - 10 yrs) & 66.00 & 25.78 & 2.31 & 25.76 \\
\hline Group 3 (11 - 15 yrs) & 25.00 & 9.76 & 1.71 & 44.00 \\
\hline Group 3 (> 15 yrs) & 11.00 & 4.30 & 0.67 & 18.18 \\
\hline \multicolumn{5}{|l|}{ League } \\
\hline Singles & 85.00 & 33.20 & 2.57 & 21.18 \\
\hline Doubles & 15.00 & 5.86 & 1.76 & 20.00 \\
\hline Both & 156.00 & 60.93 & 1.92 & 12.18 \\
\hline \multicolumn{5}{|l|}{ Warm up group } \\
\hline Group 1 (No Warm Up) & 35.00 & 13.67 & 2.65 & 25.71 \\
\hline Group 2 (< 5 minutes) & 111.00 & 43.36 & 2.27 & 10.81 \\
\hline Group 3 (5 - 10 minutes) & 73.00 & 28.51 & 2.13 & 16.44 \\
\hline Group 4 (> 10 minutes) & 37.00 & 13.67 & 1.54 & 18.92 \\
\hline \multicolumn{5}{|l|}{ Playing lessons } \\
\hline Playing lesson group & 187.00 & 73.05 & 2.10 & 16.58 \\
\hline No playing lessons, group & 69.00 & 26.95 & 2.48 & 13.04 \\
\hline
\end{tabular}

Injury: Out of 256 respondents, 35 responded that they had tennis related injury in the past 1 year that had kept them out of play for 7 days or more. The total number of the game injuries was 40 in 35 respondents.

Gender and Injury: Out of the total 35 injured players, 26 were males (26.28 years) and 9 were females (20.62 years). The numbers of non-injured male players were 147 and that of female players was 74 . 
Hours of Play per Year and Injury: Injured players had a mean of 745.33 hours of play in a year as compared to 478.87 hours for non-injured players. There was a significant difference in playing hours/year between injured and non-injured players.

Warm Up: It was found that 35 of the total subjects performed no warm up before the game however, 111 responded that they did warm up for less than 5 minutes, 75 respondents for 5-10 minutes and 37 responded that they did warm up for more than 10 minutes.

Location and Type of Injury: The study found that there were 40 reported injuries in 35 injured players, including 19 traumatic and 21 overuse injuries. The number of injuries in decreasing order was: Elbow-12 injuries (29\%), Wrist-09 injuries (22\%), Ankle-07 injuries (18\%), Shoulder-04 injuries (10\%), Knee-03 injuries, Calf-02 injuries, Thigh-02 injuries Foot-01 injury.

When trauma and overuse injuries were compared between upper and lower limbs, it was found that there were more overuse injuries in upper limbs as compared to the lower limbs and more traumatic injuries in lower limbs as compared to the upper limbs.

Injury Aggravating Strokes: Most respondents responded that backhand was the most injury aggravating strokes for elbow injuries (06 subjects). For wrist the most aggravating stroke was forehand.

Treatment: Out of the 35 injured players, 11 persons reported rest/medicine as their treatment, 06 went for physiotherapy and rest 18 chose a combination of 02 or more of rest/medicine, physiotherapy and surgery.

Timeout of competition: The study found that the average time out of competition for injured players was 29.3 days.

Recurrence: It was found that among the 35 injured players 18 were injured for the first time and 17 had reported recurrent injuries.

\section{Discussion}

The present study 256 tennis players included 173 males and 83 females were participated over a period of six months at various national tennis sports complexes. Current survey reported a total of 40 injuries (in 35 injured players) during the past one year, which kept them out of play for at least one week. The rest of 221 respondents reported no injury during the past one year. The present study defined, incidence as the number of injuries per 1000 hours, whereas, prevalence was defined as the number of injuries per 100 athletes. This study found an overall injury incidence of 2.18 injuries/1000 tennis playing hours. The prevalence of injury was found to be 15.62 injuries/100 players. Supported by a previous study done by Jayanthi et al. (2005) were reported incidence of 3.04 injuries per 1000 hours played and a prevalence of 52.9 injuries per 100 players on recreational tennis players. Although they studied an older population (mean age 46.9 years), as compared to the present study where the mean age was 22.67 years. Pluim, (2006) in a systematic review on tennis injuries, found that there was a great variation in the reported incidence rate of tennis injuries. Injury incidence varied from 0.05 to 2.9 injuries per player per year. Per hour of play, the reported incidence varied from 0.04 injuries/1000 hours to 3.0 injuries/1000 hours.
The incidence and prevalence of injury in male tennis players were 2.29 and 16.76, respectively. However the values in female tennis players were 1.91 and 13.25, respectively. This difference between the incidence of injury in males and females was statistically insignificant. This was consistent with various previous studies. Men and women play tennis in a similar manner and probably have comparable periods of activity and inactivity (Lanese et al. 1990). The present results also coincidence with the previous study, which also found that there was no significant difference between injury rates for male and female recreational tennis players in a similar study (Jayanthi et al. 2005).

Injury incidence in age groups $<16$ yrs, 16-26 yrs, and $>26$ yrs were found to be 2.35, 2.01 and 2.31, respectively, their difference was statistically insignificant. Injury prevalence in these age groups was also observed $6.35,16.00$ and 21.50 respectively. Pluim et al.(2006)in a systematic review of tennis injuries also studied the occurrence, etiology, prevention and stated that based on previous studies, in junior players injury severity was significantly less. The study reported that injury risk in tennis has been shown to gradually increasing with age, from 0.01 injuries per player per year in the 6-12 years of age group to 0.5 injuries per player per year in those over 75 years of age. Moreover Jayanthi et al.(2005)also observed in their study on skill related injury pattern in recreational tennis players found that the incidence and prevalence of tennis injuries were more in older age groups $45-55$ yrs and $>55$ years, as compared to younger age groups $<45$ years (mean age of all players was 46.9 yrs).

Players were divided into three skill groups: Group 1 (with ITN rating 1, 2 or 3), Group 2 (with ITN rating 4, 5 or 6) and Group 3 (with ITN rating 7, 8, 9 or 10). When prevalence was calculated, it was found that Group 1 had higher (42.22), Group 2 had moderate (13.67) and Group 3 had low (2.78) injury prevalence. It was found that injury prevalence was less among the players with low tennis rating, or higher ranked players were more vulnerable to injuries. For different skill level groups, injury incidence was as follows: Group $1=1.98$, Group 2 $=2.26$ and Group $3=2.94$. The difference was statistically insignificant. Jayanthi et al.(2005) in their study entitled skill level related injuries in recreational competition tennis players found that level had no effect on overall injury rates in recreational league tennis players.

With regard to warm up duration, respondents were divided into 4 groups based on duration into 'No WarmUp' (Group 1), up to 5 minutes (Group 2), 5-10 minutes (Group 3) and more than 10 minutes (Group 4).The incidence of injuries in Groups 1, 2, 3 and 4 was 2.65, $2.27,2.13$ and 1.54, respectively. This clearly showed that warm up decreased the incidence of injury, with highest incidence in those who did no warm up. It was found to be statistically insignificant. Prevalence of injury in these groups was 25.71, 10.81, 16.44 and 18.92, respectively. This showed that the group with no warm up had a maximum prevalence of injury. However, lowest prevalence of 10.81 in Group 2 could be either due to the influence of other factors or due to high number of total subjects in this group (111 subjects).

The study found that there were 40 reported injuries in 35 injured players, including 19 traumatic and 21 overuse 
injuries. The number of injuries in decreasing order was: Elbow-12 injuries (29\%), Wrist-09 injuries (22\%), Ankle07 injuries (18\%), Shoulder-04 injuries (10\%), knee-03 injuries, calf-02 injuries, thigh-02 injuries and foot-01 injury.

Our study result supports the common trends similar to previously examine populations. Sell et al. (2014) reported that muscle or tendon injuries were the most common type of acute injury. However, there were differences in injury location trends compared to previous research, suggesting that further research in this elite-level population is warranted. Sell et al. (2014) reported the rate of lower limb injuries was significantly higher than upper limb and trunk injuries. The ankle, followed by the wrist, knee, foot/toe and shoulder/clavicle were the most common injury sites.

Alberto et al. 2009, reported the imbalance between the power of the strokes and the level of physical conditioning, which includes coordination, power, strength, speed, endurance and flexibility, is responsible for negative adaptive changes that may determine the injury pattern. Jayanthi et al.(2005), in their study found the following injury pattern Elbow (20\%), Shoulder (15\%), Knee (12\%), back (10\%), Ankle (8\%), Foot (8\%), Wrist (6\%), Calf (5\%), Thigh (5\%), Lower leg (1\%) and other (3\%). However, the target population of their study was comparatively older (recreational population) as compared to our study. In our study of Indian population, we found more cases of wrist injuries, but we did not encounter any back pain case.

When the total number of traumatic and overuse injuries were compared, the study showed that overuse injuries were slightly more than traumatic. When the type of injuries was compared for upper and lower limbs, there were more overuse injuries in upper limbs as compared to lower limbs. On the other hand, there were more traumatic injuries in lower limbs as compared to the upper limbs. It is supported by a recent survey in tennis by Abrams et al. (2012) observed that tennis sports create specific demands on the musculoskeletal system, with acute injuries, such as ankle sprains, being more frequent in the lower extremity while chronic overuse injuries, such as lateral epicondylitis, are more common in the upper extremity in the recreational player and shoulder pain more common in the high-level player. However Maffulli et al. (2005), in their study on long term sport involvement and sport injury rate in elite young athletes found that tennis players had significantly more upper limb injuries, soccer players had significantly lower limb injuries and gymnasts had significantly more back injuries than other sports. Alberto et al. (2009), found that traumatic injuries occur more frequently in the lower extremities while chronic injuries are equally distributed among upper and lower extremities.

Pluim et al. (2006) observed in a systematic review of published reports for Tennis Injuries: Occurrence, Etiology and Prevention and found that four of six studies reported more acute than chronic injuries. Most acute injuries occurred in the lower extremities, whereas more chronic injuries were located in upper extremities. Kibler et al.(2005), on tennis injuries mentioned that the most common types of injury in young tennis players are micro trauma related overuse injuries, particularly to the upper extremity. Consistent with many published studies on tennis players, the most common injury in this study was elbow, followed by wrist, ankle, shoulder, knee, etc. Pluim et al.(2006) on tennis injuries stated that incidence and prevalence rates for tennis elbow were quite high, with reported incidence varying from $9 \%$ to $35 \%$ and prevalence varying from $14 \%$ to $41 \%$.

Kibler et al (2005) reported in a research on tennis injury stated that lateral epicondylitis, medial epicondylitis, and injury to the medial epicondylar apophyseal plate in skeletally immature players are common injuries about the elbow seen in tennis players. These injuries are associated with chronic repetitive overload. Lateral epicondylitis occurs more frequently in recreational tennis players. These injuries are associated with chronic repetitive overload.

The study found that the average time out of competition for injured players was 29.3 days. Kibler et al. (2005) stated that unfortunately, with so few epidemiological studies, including no studies looking specifically at the relative distribution of injuries based on time lost from tennis, no meaningful conclusions can be based on existing literature regarding time loss from play.

It was found that among the 35 injured players 18 were injured for the first time and 17 had reported recurrent injuries. Chard et al.(1987) stated that continuing to play once an injury occurs and not heeding physical warning of impending injury needs to be discouraged. It is possible that at least some of the not inconsiderable number of patients with a past history of injury to an area may have avoided further problems with care and attention to fitness of that part of the body.

In addition, player-specific factors, such as age, sex, volume of play, skill level, racquet properties and grip positions as well as the effect of playing surface on the incidence and prevalence of injury is reported. However, there were differences in injury location trends compared to previous research, suggesting that further research in this elite-level population is warranted. Finally, recommendations for standardization of future epidemiological studies on tennis injuries are made in order to be able to more easily compare results of future investigations.

\section{Conclusion}

The current study describes the prevalence, incidence, and pattern of injuries in tennis players in Indian context. Prevalence and incidence have been identified for different intrinsic and extrinsic factors, such as different skill level, warm-up duration, gender, age group and tennis experience. Tennis players sustain more overuse injuries in upper limbs and more acute injuries in lower limbs. These findings reinforce the continuing need for improved education of people undertaking this sport to try to reduce the number of injuries that may result, to limit their severity and reduce recurrence of injuries in tennis which has such a wide appeal to a large age range in general population.

\section{References}

[1] Pluim BM, Staal JB, Windler GE, Jayanthi N. Tennis injuries: occurrence, etiology, and prevention; Br J Sports MED; 2006; 40: 415-423. 
[2] Kavocs MS; Tennis physiology. Sports Medicine. 2007; 37(3): 189-198.

[3] All India Tennis Association (AITA), www.aitatennis.com. 2010 Retrieved on January 5, 2010, 02:30 p.m.

[4] Alberto ST et al. Wrist injuries in non-professional tennis players: relationship with different grips. American journal of sports medicine. 2009(37); 760-767.

[5] Dekker R et al. Long-term outcome of sport injuries: results after inpatient treatment. ClinRehabil. 2003; 17: 480-487.

[6] Maquirriain J, Ghisi JP. The incidence and distribution of stress fracture in elite tennis players. British Journal of Sports Medicine. 2006; 40: 454-459.

[7] Bylak J, Hutchinson MR; Common sports injuries in young tennis players. Sports medicine. 1998; 26(2): 119-132.

[8] Adirim TA, Cheng TL. Overview of injuries in the young athlete. Sports Med. 2003; 33(1): 75-81.

[9] Kibler WB, Safran M. Tennis Injuries; Individual sports. Med Sports Sci. 2005; 48:120-137.
[10] Brooks JHM and Fuller CW. The influence of methodological issues on the result and conclusions from epidemiological studies of sports injuries. Sports Med. 2006; 36(6): 459-472.

[11] Jayanthi $\mathrm{N}$ et al. Skill-level related injuries in recreational competition tennis players. Medicine \& Science in Tennis.2005; 10(1): 12-15.

[12] Lanese RR et al. Injury and disability in matched men's and women's intercollegiate sports. American journal of public health. 1990; 80(12):1459-1462.

[13] Sell K, Hainline B, Yorio M, et al. Injury trend analysis from the US Open Championships between 1994 and 2009. Br J Sports Med. 2014; 48: 546-51.

[14] Abrams GD, Renstrom PA, Safran MR. Epidemiology of musculoskeletal injury in the tennis player. British journal of sports medicine. 2012; 46(7): 492-498.

[15] Maffulli N et al. Long term sport involvement and sport injury rate in elite young athletes; Arch. Dis. Child. 2005; 90: 525-527.

[16] Chard MD and Lachmann SM. Racquet sports- patterns of injury presenting to a sports injury clinic. British journal of sports medicine. 1987; (21): 150-153. 